\title{
Linear $k$-power Preservers on Tensor Products of Matrices
}

\author{
Le Yan $^{1} \&$ Yang Zhang ${ }^{1}$ \\ ${ }^{1}$ School of Mathematics, Northeast Forestry University, Harbin, China \\ Correspondence: Yang Zhang, School of Mathematics, Northeast Forestry University, Harbin, 150040, China. \\ E-mail: zhangyangmath@163.com
}

Received: October 30, 2020 Accepted: November 24, 2020 Online Published: November 30, 2020

doi:10.5539/jmr.v12n6p110 URL: https://doi.org/10.5539/jmr.v12n6p110

\begin{abstract}
Invariants and the study of the map preserving a certain invariant play vital roles in the study of the theoretical mathematics. The preserver problems are the researches on linear operators that preserve certain invariants between matrix sets. Based on the result of linear $k$-power preservers on general matrix spaces, in terms of the advantages of matrix tensor products which is not limited by the size of matrices as well as the immense actual background, the study of the structure of the linear $k$-power preservers on tensor products of matrices is essential, which is coped with in this paper. That is to characterize a linear map $f: M_{m_{1} \cdots m_{l}} \rightarrow M_{m_{1} \cdots m_{l}}$ satisfying $f\left(X_{1} \otimes \cdots \otimes X_{l}\right)^{k}=f\left(\left(X_{1} \otimes \cdots \otimes X_{l}\right)^{k}\right)$ for all $X_{1} \otimes \cdots \otimes X_{l} \in M_{m_{1} \cdots m_{l}}$.
\end{abstract}

Keywords: linear preserver, $k$-power, tensor product, matrix

\section{Introduction}

Suppose $F$ is a field of $c h F=0$ and $M_{m}$ is a linear space of all $m \times m$ matrices over $F$. Suppose $l \geq 1, k, r \geq 2$ as well as $m_{1}, \cdots, m_{l} \geq 2$ are positive integers. Let $P_{m}^{2}$ and $P_{m}^{r}$ be subspaces of $M_{m}$ consisting of all idempotent and $r$-potent matrices respectively, i.e. $P_{m}^{2}=\left\{A \in M_{m}: A^{2}=A\right\}$ and $P_{m}^{r}=\left\{A \in M_{m}: A^{r}=A\right\}$. For $A \in M_{m}$ and $B \in M_{n}$, we denote by $A \otimes B \in M_{m n}$ their tensor (Kronecker) products. Generally, we denote by $M_{m_{1}} \otimes \cdots \otimes M_{m_{l}} \cong M_{m_{1} \cdots m_{l}}$.

We begin with presenting a series of definitions that we shall use throughout the paper. First of all, Let $\varphi: M_{m_{1} \cdots m_{l}} \rightarrow$ $M_{m_{1} \cdots m_{l}}$ be a linear map:

1. $\varphi$ is an idempotent preserver if $A_{1} \otimes \cdots \otimes A_{l} \in P_{m_{1} \cdots m_{l}}^{2} \Rightarrow \varphi\left(A_{1} \otimes \cdots \otimes A_{l}\right) \in P_{m_{1} \cdots m_{l}}^{2}$;

2. $\varphi$ is a $r$-potent preserver if $A_{1} \otimes \cdots \otimes A_{l} \in P_{m_{1} \cdots m_{l}}^{r} \Rightarrow \varphi\left(A_{1} \otimes \cdots \otimes A_{l}\right) \in P_{m_{1} \cdots m_{l}}^{r}$;

3. $\varphi$ is a $k$-power preserver if $\varphi\left(X_{1} \otimes \cdots \otimes X_{l}\right)^{k}=\varphi\left(\left(X_{1} \otimes \cdots \otimes X_{l}\right)^{k}\right), \forall X_{1} \otimes \cdots \otimes X_{l} \in M_{m_{1} \cdots m_{l}}$.

Then we call a linear map $\pi$ on $M_{m_{1} \cdots m_{l}}$ canonical, if $\pi$ maps $X_{1} \otimes \cdots \otimes X_{l}$ to $\tau_{1}\left(X_{1}\right) \otimes \cdots \otimes \tau_{l}\left(X_{l}\right)$, where $\tau_{k}: M_{m_{k}} \rightarrow M_{m_{k}}$ is either the identity map $X \mapsto X$ or the transposition map $X \mapsto X^{t}, k=1, \cdots, l$.

This paper is to determine the structure of linear $k$-power preservers on tensor products of matrices. The real motivation for our work derived from linear preserver problems which have become the major topic in the research of matrix theories. Frobenius (Frobenius, 1897) characterized a linear map preserving the determinant function. Chongguang (Chongguang, 1999) showed the linear maps preserving idempotence on matrix modules over some rings. Xian (Xian \& Chongguang, 2006) characterized that a linear k-power preserver between matrix spaces whose statement as follows: a linear map $f: M_{n}(F) \rightarrow M_{m}(F)$ with $c h F>k(k \in Z, k \geq 2)$ or $c h F=0$ satisfies $f(X)^{k}=f\left(X^{k}\right), \forall X \in M_{n}$ if and only if there are two nonnegative integers $p_{1}, p_{2}$ satisfying $m \geq\left(p_{1}+p_{2}\right) n$, and a nonsingular matrix $R \in M_{m}(F)$ such that $f$ has the form

$$
X \mapsto R\left[\left(X \otimes G_{1}\right) \oplus\left(X \otimes G_{2}\right) \oplus 0\right] R^{-1}, \forall X \in M_{n}(F)
$$

where $G_{i} \in M_{p_{i}}(F)$ is $k$-potent whenever $p_{i} \neq 0, i=1,2$. Baodong (Baodong, Jinli \& Ajda, 2015) determined the structure of linear maps preserving idempotents of tensor products of matrices, which is described as a linear map $\varphi: M_{m_{1} \cdots m_{l}} \rightarrow M_{n}$ preserves idempotents of tensor products of matrices if and only if either $\varphi=0$ or $n=m_{1} \cdots m_{l}$ and there is an invertible matrix $Q \in M_{n}$ and a canonical map $\pi$ on $M_{m_{1} \cdots m_{l}}$ such that $\varphi$ has the form $\varphi\left(A_{1} \otimes \cdots \otimes A_{l}\right)=Q \pi\left(A_{1} \otimes \cdots \otimes A_{l}\right) Q^{-1}, \forall A_{k} \in$ $M_{k}, k=1, \cdots, l$.

The purpose of this article is to characterize the linear preserver problems on tensor products of matrix spaces which not only enriches the theories of linear preserver problems, but is served as the theoretical basis of quantum information 
science. The matrix tensor product is an operation defined between two matrices of arbitrary size, which is not limited by the number of rows or columns. Therefore, the matrix tensor product has the characteristics of a wide application range. In addition, the requirement of tensor products is added to the linear preserver problems, which reduces the constraint on mapping and makes the conclusion more general. For more results on preserver problems, we refer the reader to Ginhor, Constantin \& Xian (Ginhor \& Minghuat, 1992; Constantin, 2019; Xian, Xioamin \& Chongguang, 2007 ).

\section{Preliminary Results}

Before verifying our main theorems, let us present a string of auxiliary results which we will need in the sequel.

lemma 1 Let $f: M_{m_{1} \cdots m_{l}} \rightarrow M_{m_{1} \cdots m_{l}}$ be a linear $k$-power preserver on tensor products of matrices. Suppose $A_{1} \otimes \cdots \otimes A_{l}$ and $B_{1} \otimes \cdots \otimes B_{l}$ are orthogonal idempotent matrices. Then $f\left(A_{1} \otimes \cdots \otimes A_{l}\right)$ and $f\left(B_{1} \otimes \cdots \otimes B_{l}\right)$ are orthogonal.

Proof Suppose $A_{1} \otimes \cdots \otimes A_{l}$ and $B_{1} \otimes \cdots \otimes B_{l}$ are orthogonal idempotent matrices, i.e.

$$
\left(A_{1} \otimes \cdots \otimes A_{l}\right)\left(B_{1} \otimes \cdots \otimes B_{l}\right)=\left(B_{1} \otimes \cdots \otimes B_{l}\right)\left(A_{1} \otimes \cdots \otimes A_{l}\right)=0
$$

Combining with the definition of $f$, we can obtain that for $\forall t \in\langle k\rangle$,

$$
\begin{aligned}
f\left(\left(A_{1} \otimes \cdots \otimes A_{l}+t\left(B_{1} \otimes \cdots \otimes B_{l}\right)\right)^{k}\right) & =f\left(\left(A_{1} \otimes \cdots \otimes A_{l}\right)^{k}\right)+t^{k} f\left(\left(B_{1} \otimes \cdots \otimes B_{l}\right)^{k}\right) \\
& =f\left(A_{1} \otimes \cdots \otimes A_{l}\right)^{k}+t^{k} f\left(B_{1} \otimes \cdots \otimes B_{l}\right)^{k}
\end{aligned}
$$

and

$$
\begin{aligned}
& f\left(A_{1} \otimes \cdots \otimes A_{l}+t\left(B_{1} \otimes \cdots \otimes B_{l}\right)\right)^{k} \\
= & \left(f\left(A_{1} \otimes \cdots \otimes A_{l}\right)+t f\left(B_{1} \otimes \cdots \otimes B_{l}\right)\right)^{k} \\
= & f\left(A_{1} \otimes \cdots \otimes A_{l}\right)^{k}+t\left[\sum_{p=0}^{k-1} f\left(A_{1} \otimes \cdots \otimes A_{l}\right)^{p} f\left(B_{1} \otimes \cdots \otimes B_{l}\right) f\left(A_{1} \otimes \cdots \otimes A_{l}\right)^{k-1-p}\right]+\cdots \\
& +t^{k-1}\left[\sum_{p=0}^{k-1} f\left(B_{1} \otimes \cdots \otimes B_{l}\right)^{p} f\left(A_{1} \otimes \cdots \otimes A_{l}\right) f\left(B_{1} \otimes \cdots \otimes B_{l}\right)^{k-1-p}\right]+t^{k} f\left(B_{1} \otimes \cdots \otimes B_{l}\right)^{k}
\end{aligned}
$$

By comparing coefficients, we derive that the coefficients of $t^{i}(i=1, \cdots, k-1)$ are zero. Let

$$
C \triangleq \sum_{p=0}^{k-1} f\left(A_{1} \otimes \cdots \otimes A_{l}\right)^{p} f\left(B_{1} \otimes \cdots \otimes B_{l}\right) f\left(A_{1} \otimes \cdots \otimes A_{l}\right)^{k-1-p}=0
$$

then

$$
\begin{aligned}
& f\left(A_{1} \otimes \cdots \otimes A_{l}\right)^{k} f\left(B_{1} \otimes \cdots \otimes B_{l}\right) \\
= & f\left(A_{1} \otimes \cdots \otimes A_{l}\right) f\left(A_{1} \otimes \cdots \otimes A_{l}\right)^{k-1} f\left(B_{1} \otimes \cdots \otimes B_{l}\right) \\
= & f\left(A_{1} \otimes \cdots \otimes A_{l}\right)\left(C-\sum_{p=0}^{k-2} f\left(A_{1} \otimes \cdots \otimes A_{l}\right)^{p} f\left(B_{1} \otimes \cdots \otimes B_{l}\right) f\left(A_{1} \otimes \cdots \otimes A_{l}\right)^{k-1-p}\right) \\
= & -\sum_{p=1}^{k-1} f\left(A_{1} \otimes \cdots \otimes A_{l}\right)^{p} f\left(B_{1} \otimes \cdots \otimes B_{l}\right) f\left(A_{1} \otimes \cdots \otimes A_{l}\right)^{k-p} \\
= & \left(-C+f\left(B_{1} \otimes \cdots \otimes B_{l}\right) f\left(A_{1} \otimes \cdots \otimes A_{l}\right)^{k-1}\right) f\left(A_{1} \otimes \cdots \otimes A_{l}\right) \\
= & f\left(B_{1} \otimes \cdots \otimes B_{l}\right) f\left(A_{1} \otimes \cdots \otimes A_{l}\right)^{k}
\end{aligned}
$$

Since $A_{1} \otimes \cdots \otimes A_{l} \in P_{m_{1} \cdots m_{l}}^{k}$ following from $A_{1} \otimes \cdots \otimes A_{l} \in P_{m_{1} \cdots m_{l}}^{2}$ and $f$ is a $k$-power preserver, we derive that

$$
f\left(A_{1} \otimes \cdots \otimes A_{l}\right)^{k}=f\left(\left(A_{1} \otimes \cdots \otimes A_{l}\right)^{k}\right)=f\left(A_{1} \otimes \cdots \otimes A_{l}\right)
$$

Then

$$
f\left(A_{1} \otimes \cdots \otimes A_{l}\right) f\left(B_{1} \otimes \cdots \otimes B_{l}\right)=f\left(B_{1} \otimes \cdots \otimes B_{l}\right) f\left(A_{1} \otimes \cdots \otimes A_{l}\right)
$$

This, together with (1), implies that

$$
\begin{aligned}
f\left(A_{1} \otimes \cdots \otimes A_{l}\right) f\left(B_{1} \otimes \cdots \otimes B_{l}\right) & =f\left(A_{1} \otimes \cdots \otimes A_{l}\right)^{k} f\left(B_{1} \otimes \cdots \otimes B_{l}\right) \\
& =f\left(A_{1} \otimes \cdots \otimes A_{l}\right) f\left(A_{1} \otimes \cdots \otimes A_{l}\right)^{k-1} f\left(B_{1} \otimes \cdots \otimes B_{l}\right) \\
& =0
\end{aligned}
$$


i.e. $f\left(A_{1} \otimes \cdots \otimes A_{l}\right)$ and $f\left(B_{1} \otimes \cdots \otimes B_{l}\right)$ are orthogonal.

lemma 2 Suppose $X_{1} \otimes \cdots \otimes X_{l} \in M_{m_{1} \cdots m_{l}}$. Then there exist invertible matrices $Q, D_{i}(i=1, \cdots, l)$ and a nilpotent matrix $N$ such that

$$
X_{1} \otimes \cdots \otimes X_{l}=Q\left(\left(D_{1} \otimes \cdots \otimes D_{l}\right) \oplus N\right) Q^{-1}
$$

proof The proof is proceeded by the induction on $l$.

The case $l=1$ is just Theorem A.0.4 in Xian (Xian, Xiaomin \& Chongguang, 2007). Then we assume that the statement holds true for $l=m-1$, i.e. there exist invertible matrices $Q_{1}, D_{i}(i=1, \cdots, m-1)$ and a nilpotent matrix $N_{1}$ such that

$$
X_{1} \otimes \cdots \otimes X_{m-1}=Q_{1}\left(\left(D_{1} \otimes \cdots \otimes D_{m-1}\right) \oplus N_{1}\right) Q_{1}^{-1}
$$

Next we will show that the conclusion is true for $l=m$ as well.

Assume that there remain invertible matrices $P, D_{m}$ and a nilpotent matrix $N_{0}$ such that

$$
X_{m}=P\left(D_{m} \oplus N_{0}\right) P^{-1}
$$

Then

$$
\begin{aligned}
X_{1} \otimes \cdots \otimes X_{m-1} \otimes X_{m} & =\left[Q_{1}\left(\left(D_{1} \otimes \cdots \otimes D_{m-1}\right) \oplus N_{1}\right) Q_{1}^{-1}\right] \otimes\left[P\left(D_{m} \oplus N_{0}\right) P^{-1}\right] \\
& =\left(Q_{1} \otimes P\right)\left[\left(\left(D_{1} \otimes \cdots \otimes D_{m-1}\right) \oplus N_{1}\right) \otimes\left(D_{m} \oplus N_{0}\right)\right]\left(Q_{1}^{-1} \otimes P^{-1}\right)
\end{aligned}
$$

By a straightforward computation, we can get an invertible matrix $T$ such that

$$
\begin{aligned}
& \left(\left(D_{1} \otimes \cdots \otimes D_{m-1}\right) \oplus N_{1}\right) \otimes\left(D_{m} \oplus N_{0}\right) \\
= & T\left[\left(D_{1} \otimes \cdots \otimes D_{m-1} \otimes D_{m}\right) \oplus\left(D_{1} \otimes \cdots \otimes D_{m-1} \otimes N_{0}\right) \oplus\left(N_{1} \otimes D_{m}\right) \oplus\left(N_{1} \otimes N_{0}\right)\right] T^{-1}
\end{aligned}
$$

Let $N \triangleq\left(D_{1} \otimes \cdots \otimes D_{m-1} \otimes N_{0}\right) \oplus\left(N_{1} \otimes D_{m}\right) \oplus\left(N_{1} \otimes N_{0}\right)$ and $Q \triangleq\left(Q_{1} \otimes P\right) T$, we conclude that

$$
X_{1} \otimes \cdots \otimes X_{m-1} \otimes X_{m}=Q\left[\left(D_{1} \otimes \cdots \otimes D_{m}\right) \oplus N\right] Q^{-1}
$$

where $N$ is nilpotent.

lemma 3 Suppose $A_{1} \otimes \cdots \otimes A_{l} \in P_{m_{1} \cdots m_{l}}^{r}$ with $R\left(A_{1} \otimes \cdots \otimes A_{l}\right)=R\left(A_{1}\right) \cdots R\left(A_{l}\right)=s_{1} \cdots s_{l} \geq 1$. Then exist invertible matrices $Q, D_{i}(i=1, \cdots, l)$ such that

$$
A_{1} \otimes \cdots \otimes A_{l}=Q\left[\left(D_{1} \otimes \cdots \otimes D_{l}\right) \oplus 0\right] Q^{-1}
$$

Proof Using lemma 2, there exist invertible matrices $Q, D_{i}(i=1, \cdots, l)$ and a nilpotent matrix $N$ such that

$$
A_{1} \otimes \cdots \otimes A_{l}=Q\left[\left(D_{1} \otimes \cdots \otimes D_{l}\right) \oplus N\right] Q^{-1}
$$

Since $N^{r}=N$ which follows from $A_{1} \otimes \cdots \otimes A_{l} \in P_{m_{1} \cdots m_{l}}^{r}$, we derive that

$$
N=N^{r}=N^{r-2} \cdot N^{2}=N^{r-2} \cdot 0=0
$$

\section{Main Results}

Theorem 1 Suppose $f: M_{m_{1} \cdots m_{l}} \rightarrow M_{m_{1} \cdots m_{l}}$ is a linear map such that

(a) $f\left(A_{1} \otimes \cdots \otimes A_{l}\right) \in P_{m_{1} \cdots m_{l}}^{r}$ for $A_{1} \otimes \cdots \otimes A_{l} \in P_{m_{1} \cdots m_{l}}^{2}$;

(b) $f\left(C_{1} \otimes \cdots \otimes C_{l}\right)$ and $f\left(D_{1} \otimes \cdots \otimes D_{l}\right)$ are orthogonal for any orthogonal idempotent matrices $C_{1} \otimes \cdots \otimes C_{l}$ and $D_{1} \otimes \cdots \otimes D_{l}$.

Then either $f=0$ or there exist an invertible matrix $T \in M_{m_{1} \cdots m_{l}}$, a canonical map $\pi$ on $M_{m_{1} \cdots m_{l}}$ and a scalar $\lambda \in F$ with $\lambda^{r-1}=1$ such that

$$
f: X_{1} \otimes \cdots \otimes X_{l} \longmapsto \lambda T \pi\left(X_{1} \otimes \cdots \otimes X_{l}\right) T^{-1}, \forall X_{1} \otimes \cdots \otimes X_{l} \in M_{m_{1} \cdots m_{l}}
$$


Proof For any $A_{1} \otimes \cdots \otimes A_{l} \in P_{m_{1} \cdots m_{l}}^{2}$, it is clear that $A_{1} \otimes \cdots \otimes A_{l}$ and $I_{m_{1} \cdots m_{l}}-A_{1} \otimes \cdots \otimes A_{l}$ are orthogonal idempotent matrices. Using $(b)$, we can obtain that $f\left(A_{1} \otimes \cdots \otimes A_{l}\right)$ and $f\left(I_{m_{1} \cdots m_{l}}-A_{1} \otimes \cdots \otimes A_{l}\right)$ are orthogonal. It follows from the linearity of $f$ that

$$
f\left(A_{1} \otimes \cdots \otimes A_{l}\right)^{2}=f\left(A_{1} \otimes \cdots \otimes A_{l}\right) f\left(I_{m_{1} \cdots m_{l}}\right)=f\left(I_{m_{1} \cdots m_{l}}\right) f\left(A_{1} \otimes \cdots \otimes A_{l}\right)
$$

In terms of the invertibility of $f\left(I_{m_{1} \cdots m_{l}}\right)$, then we will divide it into the following two cases.

Case 1: Suppose $f\left(I_{m_{1} \cdots m_{l}}\right)$ is nonsingular, i.e. $R\left(f\left(I_{m_{1} \cdots m_{l}}\right)=m_{1} \cdots m_{l}\right.$

Define a map $h: M_{m_{1} \cdots m_{l}} \rightarrow M_{m_{1} \cdots m_{l}}$ by

$$
h\left(X_{1} \otimes \cdots \otimes X_{l}\right)=f\left(I_{m_{1} \cdots m_{l}}\right)^{-1} f\left(X_{1} \otimes \cdots \otimes X_{l}\right), \forall X_{1} \otimes \cdots \otimes X_{l} \in M_{m_{1} \cdots m_{l}}
$$

Combining with (2) and the linearity of $f$, we can conclude that $h$ is a linear idempotent preserver on tensor products of matrices satisfying $h\left(I_{m_{1} \cdots m_{l}}\right)=I_{m_{1} \cdots m_{l}}$. According to the main theorem in Baodong (Baodong, Jinli \& Ajda, 2015), there is an invertible matrix $T \in M_{m_{1} \cdots m_{l}}$ and a canonical map $\pi$ on $M_{m_{1} \cdots m_{l}}$ such that $h$ has the form

$$
h: X_{1} \otimes \cdots \otimes X_{l} \longmapsto T \pi\left(X_{1} \otimes \cdots \otimes X_{l}\right) T^{-1}, \forall X_{1} \otimes \cdots \otimes X_{l} \in M_{m_{1} \cdots m_{l}}
$$

Thus $f$ has the form

$$
f: X_{1} \otimes \cdots \otimes X_{l} \longmapsto f\left(I_{m_{1} \cdots m_{l}}\right) T \pi\left(X_{1} \otimes \cdots \otimes X_{l}\right) T^{-1}, \forall X_{1} \otimes \cdots \otimes X_{l} \in M_{m_{1} \cdots m_{l}}
$$

By (2), we have

$$
f\left(I_{m_{1} \cdots m_{l}}\right) T \pi\left(A_{1} \otimes \cdots \otimes A_{l}\right) T^{-1}=T \pi\left(A_{1} \otimes \cdots \otimes A_{l}\right) T^{-1} f\left(I_{m_{1} \cdots m_{l}}\right)
$$

for any $A_{1} \otimes \cdots \otimes A_{l} \in P_{m_{1} \cdots m_{l}}^{2}$.

Assume $f\left(I_{m_{1} \cdots m_{l}}\right)=T U T^{-1}$, then $U \pi\left(A_{1} \otimes \cdots \otimes A_{l}\right)=\pi\left(A_{1} \otimes \cdots \otimes A_{l}\right) U$. According to lemma 2.3 in Baodong (Baodong, Jinli \& Ajda, 2015), it is obvious that $U=\lambda I_{m_{1} \cdots m_{l}}$ where $\lambda \in F$ with $\lambda \neq 0$. Therefore $f\left(I_{m_{1} \cdots m_{l}}\right)=\lambda I_{m_{1} \cdots m_{l}}$. Combining with $I_{m_{1} \cdots m_{l}} \in P_{m_{1} \cdots m_{l}}^{2}$ and (a), we obtain that $\lambda^{r-1}=1$. Consequently, $f$ has the following form

$$
f: X_{1} \otimes \cdots \otimes X_{l} \longmapsto \lambda T \pi\left(X_{1} \otimes \cdots \otimes X_{l}\right) T^{-1}, \forall X_{1} \otimes \cdots \otimes X_{l} \in M_{m_{1} \cdots m_{l}}
$$

where $\lambda \in F$ with $\lambda^{r-1}=1$.

Case 2: Suppose $f\left(I_{m_{1} \cdots m_{l}}\right)$ is singular.

1) Suppose $f\left(I_{m_{1} \cdots m_{l}}\right)=0$.

Then we derive from (2) that

$$
f\left(A_{1} \otimes \cdots \otimes A_{l}\right)^{2}=0, \forall A_{1} \otimes \cdots \otimes A_{l} \in P_{m_{1} \cdots m_{l}}^{2}
$$

This, together with $(a)$, implies that

$$
f\left(A_{1} \otimes \cdots \otimes A_{l}\right)=f\left(A_{1} \otimes \cdots \otimes A_{l}\right)^{r}=f\left(A_{1} \otimes \cdots \otimes A_{l}\right)^{r-2} f\left(A_{1} \otimes \cdots \otimes A_{l}\right)^{2}=0
$$

Due to every matrix in $M_{m_{1} \cdots m_{l}}$ can be written as a linear combination of finitely many idempotent matrices in $M_{m_{1} \cdots m_{l}}$, one can conclude that

$$
f\left(X_{1} \otimes \cdots \otimes X_{l}\right)=0, \forall X_{1} \otimes \cdots \otimes X_{l} \in M_{m_{1} \cdots m_{l}}
$$

i.e. $f=0$.

2) Suppose $1 \leq R\left(f\left(I_{m_{1} \cdots m_{l}}\right)=s_{1} \cdots s_{l}<m_{1} \cdots m_{l}\right.$.

Using $I_{m_{1} \cdots m_{l}} \in P_{m_{1} \cdots m_{l}}^{2}$ and $(a)$, we have $f\left(I_{m_{1} \cdots m_{l}}\right) \in P_{m_{1} \cdots m_{l}}^{r}$. Furthermore, according to lemma 3 , there exist invertible matrices $Q, D_{i}(i=1, \cdots, l)$ such that

$$
f\left(I_{m_{1} \cdots m_{l}}\right)=Q\left(\left(D_{1} \otimes \cdots \otimes D_{l}\right) \oplus 0\right) Q^{-1}
$$

This, together with (2), implies that

$$
f\left(A_{1} \otimes \cdots \otimes A_{l}\right)=Q\left(g\left(A_{1} \otimes \cdots \otimes A_{l}\right) \oplus 0\right) Q^{-1}, \forall A_{1} \otimes \cdots \otimes A_{l} \in P_{m_{1} \cdots m_{l}}^{2}
$$

where

$$
g\left(I_{m_{1} \cdots m_{l}}\right)=D_{1} \otimes \cdots \otimes D_{l}
$$


and

$$
g\left(A_{1} \otimes \cdots \otimes A_{l}\right)^{2}=g\left(I_{m_{1} \cdots m_{l}}\right) g\left(A_{1} \otimes \cdots \otimes A_{l}\right)=g\left(A_{1} \otimes \cdots \otimes A_{l}\right) g\left(I_{m_{1} \cdots m_{l}}\right)
$$

Due to every matrix in $M_{m_{1} \cdots m_{l}}$ can be written as a linear combination of finitely many idempotent matrices in $M_{m_{1} \cdots m_{l}}$, one can conclude that

$$
f\left(X_{1} \otimes \cdots \otimes X_{l}\right)=Q\left(g\left(X_{1} \otimes \cdots \otimes X_{l}\right) \oplus 0\right) Q^{-1}, \forall X_{1} \otimes \cdots \otimes X_{l} \in M_{m_{1} \cdots m_{l}}
$$

where $g: M_{m_{1} \cdots m_{l}} \rightarrow M_{s_{1} \cdots s_{l}}$ is a linear map satisfying $(a)(b)(3)$. Define a map $h_{1}: M_{m_{1} \cdots m_{l}} \rightarrow M_{s_{1} \cdots s_{l}}$ by

$$
h_{1}\left(X_{1} \otimes \cdots \otimes X_{l}\right)=g\left(I_{m_{1} \cdots m_{l}}\right)^{-1} g\left(X_{1} \otimes \cdots \otimes X_{l}\right), \forall X_{1} \otimes \cdots \otimes X_{l} \in M_{m_{1} \cdots m_{l}}
$$

Combining with (3) and the linearity of $g$, we can conclude that $h_{1}$ is a linear idempotent preserver on tensor products of matrices. And according to the claim 2 in Baodong (Baodong, Jinli \& Ajda, 2015), we derive that $h_{1}=0$ when $m_{1} \cdots m_{l}>s_{1} \cdots s_{l}$. Then

$$
g\left(X_{1} \otimes \cdots \otimes X_{l}\right)=g\left(I_{m_{1} \cdots m_{l}}\right) h_{1}\left(X_{1} \otimes \cdots \otimes X_{l}\right)=0, \forall X_{1} \otimes \cdots \otimes X_{l} \in M_{m_{1} \cdots m_{l}}
$$

i.e. $g=0$. Thus $f=0$.

Theorem 2 A linear map $f: M_{m_{1} \cdots m_{l}} \rightarrow M_{m_{1} \cdots m_{l}}$ is a $k$-power preserver on tensor products of matrices if only if either $f=0$ or there exist an invertible matrix $T \in M_{m_{1} \cdots m_{l}}$, a canonical map $\pi$ on $M_{m_{1} \cdots m_{l}}$ and a scalar $\lambda \in F$ with $\lambda^{k-1}=1$ such that

$$
f: X_{1} \otimes \cdots \otimes X_{l} \longmapsto \lambda T \pi\left(X_{1} \otimes \cdots \otimes X_{l}\right) T^{-1}, \forall X_{1} \otimes \cdots \otimes X_{l} \in M_{m_{1} \cdots m_{l}} .
$$

Proof Since the sufficiency part of the main theorem is clear, we consider only the necessity part.

Let $f: M_{m_{1} \cdots m_{l}} \rightarrow M_{m_{1} \cdots m_{l}}$ be a linear $k$-power preserver on tensor products of matrices. For one thing, since $A_{1} \otimes \cdots \otimes A_{l} \in$ $P_{m_{1} \cdots m_{l}}^{2}$, we derive that $A_{1} \otimes \cdots \otimes A_{l} \in P_{m_{1} \cdots m_{l}}^{k}$. Combining with the definition of $f$, we get $f\left(A_{1} \otimes \cdots \otimes A_{l}\right) \in P_{m_{1} \cdots m_{l}}^{k}$, i.e.

$$
f\left(A_{1} \otimes \cdots \otimes A_{l}\right)^{k}=f\left(\left(A_{1} \otimes \cdots \otimes A_{l}\right)^{k}\right)=f\left(A_{1} \otimes \cdots \otimes A_{l}\right)
$$

For another, using lemma 1 , we can conclude that $f\left(C_{1} \otimes \cdots \otimes C_{l}\right)$ and $f\left(D_{1} \otimes \cdots \otimes D_{l}\right)$ are orthogonal when $C_{1} \otimes \cdots \otimes C_{l}$ and $D_{1} \otimes \cdots \otimes D_{l}$ are orthogonal idempotent matrices. Then the proof can be completed according to theorem 1 .

\section{Conclusion}

The structure of linear $k$-power preservers on tensor products of matrices is proposed in this paper. Based on this result, the structures of linear $k$-power preservers on tensor products of hermite matrices and symmetric matrices could continue to consider. Meanwhile, there remain a string of preserver problems on tensor products matrix spaces should be studied yielding to the application on quantum information science.

\section{References}

Chongguang, C. (1999). Linear maps idempotence on matrix modules over some rings. Heilongjiang Univ Natur Sci, 16(1), 1-4. Retrieved from https://kns-cnki-net-443.webvpn.nefu.edu.cn/kns8/defaultresult/index

Frobenius, G. (1897). Über die Darstellung der Endlichen Gruppen durch Linear Substitutionen. Sitzungsber Deutsch Aka Wis Berlin.. 994-1015.

Zejun, H., Shiyu, S., \& NungSing, S. (2016). Linear rank preservers of tensor products of rank one matrices. Linear Algebra Appl., (508), 255-271. https://doi-org-443.webvpn.nefu.edu.cn/10.1016/j.laa.2016.07.024

Jinli, X., Ajda, F., Baodong, Z., \& Yuting, D. (2017). Linear maps preserving r-potents of tensor products of matrices. Linear Algebra Appl, (520), 67-76. https://doi-org-443.webvpn.nefu.edu.cn/10.1016/j.laa.2017.01.016

Xian, Z., \& Chongguang, C. (2006). Linear k-power/k-potent preservers between matrix spaces. Linear Algebra Appl., (412), 373-379. https://doi-org-443.webvpn.nefu.edu.cn/10.1016/j.laa.2005.07.006

Yorick, H., \& Ajda, F. (2018). Linear maps preserving Kronecker quotients. Linear Algebra Appl, (556), 200-209. https://doi-org-443.webvpn.nefu.edu.cn/10.1016/j.laa.2018.07.021

Baodong, Z., Jinli, X., \& Ajda, F. (2015). Linear maps preserving idempotents of tensor products of matrices. Linear Algebra Appl., (470), 25-39. https://doi-org-443.webvpn.nefu.edu.cn/10.1016/j.laa.2014.01.036

Ginhor, C., \& Minghuat, L. (1992). Linear preservers on powers of matrices. Linear Algebra Appl., (162-164)515-626. https://doi-org-443.webvpn.nefu.edu.cn/10.1016/0024-3795(92)90396-R 
Constantin, C. (2019). Nonlinear determinant preserving maps on matrix algebras. Linear Algebra Appl., (583), 165-170. https://doi-org-443.webvpn.nefu.edu.cn/10.1016/j.laa.2019.09.003

Xian, Z., Xiaomin, T., \& Chongguang, C. (2007). Preserver Problems on Spaces of Matrices. Science Press, Beijing.

\section{Copyrights}

Copyright for this article is retained by the author(s), with first publication rights granted to the journal.

This is an open-access article distributed under the terms and conditions of the Creative Commons Attribution license (http://creativecommons.org/licenses/by/4.0/). 